\title{
Interdisciplinary and Inter-Functional Psychomotricity at School
}

\section{Dario Furnari}

University of Exeter, UK

Corresponding author: Dario Furnari, University of Exeter, UK. E-mail : dariofurnari@hotmail.it

Received: January 29, 2020, Accepted: February 17, 2020 Published: February 28, 2020

Keywords: Psychomotricity, Physical activity, Aerobic Mootr activity

Physical activity, which is often recommended by doctors, refers to a regular "aerobic" motor activity that can vary for each subject based on their characteristics and / or pathologies. In practice this activity can vary from walks with a sustained pace to slow running or cycling with city bikes for short journeys repeated periodically in the week or swimming $2 / 3$ times a week also regularly follow gym classes or workout programs in the gym are to be considered as a good physical activity.

Physical activity aims to improve cardio-circulatory and respiratory functions to tone muscles and improve joint movement skills.

Doing sports means dedicating oneself to a sporting discipline, constantly training to improve both physical performance and technique in athletic gestures and game patterns. Professional sport, as the word itself says, refers to athletes who, due to their genetic characteristics, have a predisposition for a discipline obtaining good performances for these characteristics, perhaps with a little luck they manage to enter the limbo of the elect and sport they make a profession often with significant economic benefits. The career of professional athlete can be more or less long, but still short compared to normal working life.
The amateur sport, is practiced by millions of people, of all ages who train constantly in a specific way for each discipline with the aim to compete in championships or competitions with athletes of the same age or category. Often they are people who continue with sports done by boys or some change completely discipline for others is a discovery in the late age of passion for sport.

-"In the sportsman at the base of a good performance, there is always good nutrition"

-"In man, at the basis of well-being there is always proper nutrition".

Our organism for life constantly needs energy. We consume energy to work, study, breathe, sleep, etc .. because each organ or apparatus is made up of billions of cells in which there are many biochemical processes, catalyzed by enzymes, that allow us to live. All this has a considerable expenditure of energy.

Every mechanical means of traveling needs energy that can be supplied, from petrol, diesel, electric power, etc. our body also needs fuel supplied by foods that, once digested, thanks to the biochemical processes mentioned above, are transformed into "ATP" (adenosine-tri-phosphate) and 
Citation: Dario Furnaril (2020) Interdisciplinary and Inter-Functional Psychomotricity at School. Journal of Physical Medicine Rehabilitation Studies \& Reports. SRC/JPMRS/103. DOI: doi.org/10.47363/JPMRS/2020(2)103

available as an energy source.

As for a race car to be able to make the engine work better and to get high performance, you need a lot of fuel and excellent quality, in the athlete "at the base of a good athletic performance there is always an excellent supply".

To improve every physical performance, it is first of all to follow with intelligence training programs and the technical diagrams that are given by the coaches, try to correct their mistakes and improve the execution of athletic gestures and keep the "engine" your body at its best of the conditions providing "petrol".

\section{Food of Excellent Quality}

"Each engine is designed to work up to certain levels, if the engine is broken, it breaks"

Every athlete has some athletic characteristics Genetically determined to overcome them means to have damages often irreparable,

"The doping substances overcome these limits, improving performance for a short period of time, with serious damage to the athlete's health".

\section{Energy}

Defined as the ability to produce work.

Here are two forms of energy that most interest us: 1) E. Mechanics 2) E. Chemistry.

1)Mechanical energy can be the expression of a tennis bat of a golf club or baseball that with their oscillating movements perform a mechanical job. The stroke, with its displacement in the frontal direction of the center of gravity can be an expression of mechanical work. The energy produced by these movements is defined in "Kinetic energy".

The mechanical work carried out by virtue of the position, how to lift a body in the opposite direction to its gravity is called "Potential energy"

2) Chemical energy is a source of potential energy In the body foods are degraded through chemical reactions with energy release, which is in turn used to synthesize other chemical compounds; once degraded, they release chemical energy that is used by skeletal muscles to perform mechanical work.

In other words, a part of the chemical energy present in foods is converted from skeletal muscles into Mechanical or Kinetic Energy.

The unit of energy measurement is the CALORY. Sports can be classified based on the use of energy in:

\section{Anaerobics Aerobics}

Anaerobics can in turn be classified into:

1) LACTACIDES that produce lactic acid

2) ALACTACIDS which do not produce lactic acid

Before discussing the biological and metabolic mechanisms that differentiate the different use of energy, we classify sports according to their energy requirements.

Aerobic Sports: "Require Energy over an extended period of time"

1. Cycling

2. Running (marathon, half marathon)

3. swimming over long distances $(800,1500) \mathrm{m}$.

4. Cross-country skiing

5. Rowing

Anaerobic Sports: "They require high energy in a short period of time'

These must be further classified based at the time of the services.

1. Alactacids are activities that have a duration of less than 2 minutes.

Fast travel with and without obstacles $(100,200,400) \mathrm{m}$

- Swimming $(50,100,200) \mathrm{m}$

- Tennis

- Volleyball

- Baseball

- Throwing (hammer, disc, javelin, weight,)

- Jump (high, long, with rod)

- Skiing (slalom) downhill

2. Lactacids are activities that last between 2-3 minutes

- Travel (800.1500) m

- Swimming $(400,800) \mathrm{m}$

- Football

- Water polo

- Basketball

- Handball

In team sports it is often difficult to make a classification because for the duration of the often brief athletic gesture that does not exceed one minute should be classified alactacids 
Citation: Dario Furnaril (2020) Interdisciplinary and Inter-Functional Psychomotricity at School. Journal of Physical Medicine Rehabilitation Studies \& Reports. SRC/JPMRS/103. DOI: doi.org/10.47363/JPMRS/2020(2)103

\section{Energy Sources}

"ATP" ready-to-use energy

ATP or adenosine triphosphate is a form of chemical energy that is ready for muscle contraction. This energy source is stored in a large amount of cells, especially those in muscle.

All forms of chemical energy, such as those taken through food must be transformed into ATP to be used.

The chemical structure of ATP is very complex but for our purpose it is sufficient to know that it is composed of a large molecular complex ADENOSINE, and three simpler complexes called phosphorus linked together by high energy bonds

\section{ADENOSINE $==\mathrm{P}==\mathrm{P}==\mathrm{P}$}

When the link with the last phosphorus breaks, a large amount of energy is released which allows the cells to do their work

\section{ADENOSINE $==\mathrm{P}==\mathrm{P}=/ /=\mathrm{P}$}

\section{Power}

- Mechanical work (contraction) is performed by muscle cells

- Conduction of nerve stimuli occurs through nerve cells

- Secretions depend on secretory cells.

Any work done by the cells requires an immediate energetic relationship that is provided by the breaking of the phosphoric bond with the release of energy.

The amount of energy per molecule of hydrolysed ATP is between $7 / 12 \mathrm{kcal}$.

\section{Mole}

Quantity of chemical substance expressed in grams equal to its molecular weight which depends on the type and number of atoms that make up the compound.

\section{Paired Reactions}

If for a muscular contraction the energy coming from the hydrolysis of the phosphoric bond of the ATP is used with the formation of ADP (adenosindiphosphate) and release of phosphorus (P) to synthesize new ATP it needs further energy and the use of the byproducts of hydrolysis ie the ADP and the $\mathrm{P}$.

The energy needed for the resynthesis derives from three different reactions that occur in the organism: two of them depend on the foods we eat the third from Fosfocreatina, a substance similar to ATP and also stored in the muscles.

The various reactions are functionally linked in such a way that the energy released by one of them is always used by the other "COUPLE REACTION"

Disability and Adaptability in the school context; The well-being of the person with educational and preventative Psychomotricity.

Psychomotor intervention is aimed primarily at patients / people in developmental age, especially children with delayed and coordination difficulties, hyperactivity and attention disorder, Autism and Generalized Developmental Disorders (DGS), psychomotor inhibition, and disorders resulting from neurological, psychological or psychiatric diseases. In this regard, the following project is aimed at all school operators, families or young mothers, students with disabilities and otherwise, for a better integration of the disabled in a socio-educational context; all this through the principle of Cooperative learning, which aims to create a non-competitive, responsibly and collaboratively play-educational context, able to promote motor and cognitive learning.

Together with the students it will be possible to pursue different objectives, which can be adapted to their specific needs. It will be important to develop body expression and non-verbal forms of communication in the case of a language difficulty, or the tactile and auditory aspects can be enhanced if disability is related to the low vision or blindness of one of the pupils, rather than the sign language in the case of deafness. In doing so, it will be possible to improve socialization within the group and promote the development of the disabled child by prolonging the time on the activities targeted to him.

\section{Knowledge Path for Teachers}

- Being able to recognize the social changes that affect the family and the consequences in the school environment;

- Know the legal elements that regulate school relations, including the organizational legal instruments

- To know and be able to interpret the relational dynamics of the developmental age and the relational dynamics in the groups;

- Knowing and knowing how to interpret the signs and Volume 2 | Issue 1 | 3 of 4 
Citation: Dario Furnaril (2020) Interdisciplinary and Inter-Functional Psychomotricity at School. Journal of Physical Medicine Rehabilitation Studies \& Reports. SRC/JPMRS/103. DOI: doi.org/10.47363/JPMRS/2020(2)103

manifestations of discomfort in its various forms

- Acquire tools for managing stressful situations due to cultural and clinical factors.

\section{Path of Knowledge for Parents}

Awareness of oneself and one's emotions through the development of emotional intelligence;

Stress management, relaxation for well-being through meditation and relaxation techniques such as autogenic training and trance dance;

Psychodynamic dialogue between parents and children through: movement - massage - music therapy - dance therapy - food education, for a harmonious and global growth of the child / child.

The project aims to carry out preventive actions for the well-being of children and young people at school, in the family and in the community.

This project addressed to three types of people, such as teachers or ata staff, parents, elementary school children and middle and high school children, poses the problem of the role of the adult both as support to the child / boy on the path to the autonomy and both in accompanying them to the discovery of their emotions, fear-anger-aggression, typical of this "delicate" and "sensitive" age.

The child revisits the phases of growth and through psychomotor play, motor sense, symbolic and socialization, represents desires, exorcises fears, resolves intrapsychic conflict situations, experiments new relationship strategies.

\section{The Project Uses the Following Methods and Techniques: Psychomotricity}

Interdisciplinary and interfunctional psychomotricity is a process of change and transformation that is carried out in groups, using body language in a spontaneous game context. It is not structured in exercises but rather in experiential sequences that the group interprets giving life to body games in full freedom. This mode is used both with children and adolescents and with adults.

The experiential groups and the psychological games functional to them are based above all on the so-called "human potential", that is on those dimensions, such as freedom, will, creativity, sociality, considered the most significant and typical of human experience.

Relational or functional psychomotility is an activity that can be practiced at any age, starting from the spatial movements of the first year of life up to the third age.

It takes place in a group and puts the body into play and, at the same time, the emotions, thoughts and memories. The relational practice allows this type of psychomotricity to adapt to each age group while maintaining a single basic methodology.

This is why parental involvement is important; to encourage the return of an inner dialogue with oneself and an intrauterine dialogue with one's own son lost over the years.

In our skin there is the memory of what we have forgotten
Copyright: @2020 Guillamón EM This is an open-access article distributed under the terms of the Creative Commons Attribution License, which permits unrestricted use, distribution, and reproduction in any medium, provided the original author and source are credited. 